\title{
On The Modified Orthogonal Frame with Curvature and Torsion in 3-Space
}

\author{
Bahaddin BUKCU* and Murat Kemal KARACAN
}

(Communicated by Erdal ÖZÜSAĞLAM)

\begin{abstract}
In this paper, we study the modified orthogonal vector fields with curvature and torsion of a space curve in Minkowski 3-Space.

Keywords: Frenet-Serret Frame, Orthogonal Frame, Modified Orthogonal Frame, Minkowski 3-Space

AMS Subject Classification (2010): Primary: 53A04 ; Secondary: 53C50; 53C40.

${ }^{*}$ Corresponding author
\end{abstract}

\section{Introduction}

In differential geometry, the Frenet-Serret formulas describe the kinematic properties of a particle moving along a continuous, differentiable curve in 3-dimensional Euclidean space $R^{3}$, or the geometric properties of the curve itself irrespective of any motion. More specifically, the formulas describe the derivatives of the so-called tangent, normal, and binormal unit vectors in terms of each other. The formulas are named after the two French mathematicians who independently discovered them: Jean Frédéric Frenet, in his thesis of 1847, and Joseph Alfred Serret in 1851 Vector notation and linear algebra currently used to write these formulas were not yet in use at the time of their discovery [1]. In ([8]), Sasai studied an orthogonal frame and obtained a formula which corresponds to the Frenet-Serret equation.

In this paper, we have generalized orthogonal frame with curvature which is obtained by T.Sasai and orthogonal frame with torsion in Minkowski 3-space.

\section{Preliminaries}

We first recall the classical fundamental theorem of space curves, i.e., curves in Euclidean 3-space $E^{3}$. Let $\alpha$ be a curve of class $C^{3}$, where $s$ is the arc-length parameter. Moreover we assume that its curvature $\kappa(s)$ does not vanish anywhere. Then there exists an orthonormal frame $\{\mathbf{t}, \mathbf{n}, \mathbf{b}\}$ which satisfies the Frenet-Serret equation

$$
\left[\begin{array}{l}
\mathbf{t}^{\prime}(s) \\
\mathbf{n}^{\prime}(s) \\
\mathbf{b}^{\prime}(s)
\end{array}\right]=\left[\begin{array}{ccc}
0 & \kappa & 0 \\
-\kappa & 0 & \tau \\
0 & -\tau & 0
\end{array}\right]\left[\begin{array}{c}
\mathbf{t}(s) \\
\mathbf{n}(s) \\
\mathbf{b}(s)
\end{array}\right],
$$

where $\mathbf{t}, \mathbf{n}$ and $\mathbf{b}$ are the tangent, principal normal and binormal unit vectors, respectively, and $\tau(s)$ is the torsion. Given a function $\kappa(s)$ of class $C^{1}$ and a continuous function $\tau(s)$, there exists a curve of class $C^{3}$ which admits an orthonormal frame $\{\mathbf{t}, \mathbf{n}, \mathbf{b}\}$ satisfying the Eq.(2.1) with given $\kappa$ and $\tau$ as its curvature and torsion, respectively. Such a curve is uniquely determined up to a motion of $E^{3}$.

Received : 05-January-2016, Accepted : 14-April-2016 
Let $\alpha_{i}(i=1,2,3)$ be coordinates in $E^{3}$. Let $\alpha(s)$ an analytic curve, where $s$ runs through some interval and $\alpha(s)$ be analytic in $s$. We assume that $\alpha$ is non-singular, i.e.

$$
\sum_{i=1}^{3}\left(\frac{d \alpha_{i}}{d s}\right)^{2}
$$

is nowhere zero. Therefore we can parametrize $\alpha$ by its arc length $s$. From now on, we only consider $\alpha$ in the following form:

$$
\alpha=\alpha(s)=\left(\alpha_{1}, \alpha_{2}, \alpha_{3}\right), s \in I
$$

where $\alpha(s)$ is analytic in $s$ and $I$ is a non-empty open interval. We assume that the curvature $\kappa(s)$ of $\alpha$ is not identically zero. Now we define an orthogonal frame $\{T, N, B\}$ as follows:

$$
T=\frac{d \alpha}{d s}, N=\frac{d T}{d s}, B=T \times N,
$$

where $T \times N$ is the vector product of $T$ and $N$. The relations between those and the classical Frenet frame $\{\mathbf{t}, \mathbf{n}, \mathbf{b}\}$ at non-zero points of $\kappa$ are

$$
\left\{\begin{array}{c}
T=\mathbf{t} \\
N=\kappa \mathbf{n} \\
B=\kappa \mathbf{b} .
\end{array}\right.
$$

Thus $N\left(s_{0}\right)=B\left(s_{0}\right)=0$ when $\kappa\left(s_{0}\right)=0$ and squares of the length of $N$ and $B$ vary analytically in $s$. By the definition of $\{T, N, B\}$ or Eq.(2.2), a simple calculation shows that

$$
\left[\begin{array}{c}
T^{\prime}(s) \\
N^{\prime}(s) \\
B^{\prime}(s)
\end{array}\right]=\left[\begin{array}{ccc}
0 & 1 & 0 \\
-\kappa^{2} & \frac{\kappa^{\prime}}{\kappa} & \tau \\
0 & -\tau & \frac{\kappa^{\prime}}{\kappa}
\end{array}\right]\left[\begin{array}{c}
T(s) \\
N(s) \\
B(s)
\end{array}\right]
$$

where a dash denotes the differentiation with respect to the arc length $s$ and

$$
\tau=\tau(s)=\frac{\operatorname{det}\left(\alpha^{\prime}, \alpha^{\prime \prime}, \alpha^{\prime \prime \prime}\right)}{\kappa^{2}}
$$

is the torsion of $\alpha$. From the Frenet-Serret equation, we know that any zero point of $\kappa^{2}$ is a removable singularity of $\tau$. The Eq.(2.3) corresponds to the Frenet-Serret equation in the classical case. Moreover, $\{T, N, B\}$ satisfies:

$$
\begin{gathered}
\langle T, T\rangle=1,\langle N, N\rangle=\langle B, B\rangle=\kappa^{2}, \\
\langle T, N\rangle=\langle T, B\rangle=\langle N, B\rangle=0
\end{gathered}
$$

where $\langle$,$\rangle denotes the inner product of E^{3}$. We note that the essential quantities in Eq.(2.3) and Eq.(2.4) are $\kappa^{2}$ and $\tau(s)$ which are analytic in $s[8]$.

The Minkowski 3-space $E_{1}^{3}$ is the Euclidean 3-space $E^{3}$ provided with the standard flat metric given by

$$
\langle,\rangle=-d x_{1}^{2}+d x_{2}^{2}+d x_{3}^{2}
$$

where $\left(x_{1}, x_{2}, x_{3}\right)$ is a rectangular coordinate system of $E_{1}^{3}$.

Since $\langle$,$\rangle is an indefinite metric, recall that a vector v$ can have one of three Lorentzian causal characters: it can be spacelike if $\langle v, v\rangle>0$ or $v=0$, timelike if $\langle v, v\rangle<0$ and null (lightlike) if $\langle v, v\rangle=0$ and $v \neq 0$.Similarly, an arbitrary curve $\alpha=\alpha(s)$ in $E_{1}^{3}$ can locally be spacelike, timelike or null (lightlike), if all of its velocity vectors $\alpha^{\prime}(s)=\mathbf{t}$ are respectively spacelike, timelike or null (lightlike). Denote by $\{\mathbf{t}, \mathbf{n}, \mathbf{b}\}$ the moving Frenet frame along the curve $\alpha(s)$ in the space $E_{1}^{3}$.For an arbitrary curve $\alpha(s)$ in the space $E_{1}^{3}$ the following Frenet formulae are given in [2-7].

If $\alpha$ is timelike curve, then the Frenet formulae read

$$
\left[\begin{array}{c}
\mathbf{t}^{\prime} \\
\mathbf{n}^{\prime} \\
\mathbf{b}^{\prime}
\end{array}\right]=\left[\begin{array}{ccc}
0 & \kappa & 0 \\
\kappa & 0 & \tau \\
0 & -\tau & 0
\end{array}\right]\left[\begin{array}{l}
\mathbf{t} \\
\mathbf{n} \\
\mathbf{b}
\end{array}\right],
$$

where

$$
\langle\mathbf{t}, \mathbf{t}\rangle=-1,\langle\mathbf{n}, \mathbf{n}\rangle=1,\langle\mathbf{b}, \mathbf{b}\rangle=1,\langle\mathbf{t}, \mathbf{n}\rangle=\langle\mathbf{n}, \mathbf{b}\rangle=\langle\mathbf{t}, \mathbf{b}\rangle=0 .
$$


If $\alpha$ is a spacelike curve with a spacelike principal normal $\mathbf{n}$, then the Frenet formulae read

$$
\left[\begin{array}{l}
\mathbf{t}^{\prime} \\
\mathbf{n}^{\prime} \\
\mathbf{b}^{\prime}
\end{array}\right]=\left[\begin{array}{ccc}
0 & \kappa & 0 \\
-\kappa & 0 & \tau \\
0 & \tau & 0
\end{array}\right]\left[\begin{array}{l}
\mathbf{t} \\
\mathbf{n} \\
\mathbf{b}
\end{array}\right],
$$

where

$$
\langle\mathbf{t}, \mathbf{t}\rangle=\langle\mathbf{n}, \mathbf{n}\rangle=1,\langle\mathbf{b}, \mathbf{b}\rangle=-1,\langle\mathbf{t}, \mathbf{n}\rangle=\langle\mathbf{n}, \mathbf{b}\rangle=\langle\mathbf{t}, \mathbf{b}\rangle=0
$$

If $\alpha$ is a spacelike curve with a spacelike binormal $\mathbf{b}$, then the Frenet formulae read

$$
\left[\begin{array}{l}
\mathbf{t}^{\prime} \\
\mathbf{n}^{\prime} \\
\mathbf{b}^{\prime}
\end{array}\right]=\left[\begin{array}{lll}
0 & \kappa & 0 \\
\kappa & 0 & \tau \\
0 & \tau & 0
\end{array}\right]\left[\begin{array}{l}
\mathbf{t} \\
\mathbf{n} \\
\mathbf{b}
\end{array}\right]
$$

where

$$
\langle\mathbf{t}, \mathbf{t}\rangle=\langle\mathbf{b}, \mathbf{b}\rangle=1,\langle\mathbf{n}, \mathbf{n}\rangle=-1,\langle\mathbf{t}, \mathbf{n}\rangle=\langle\mathbf{n}, \mathbf{b}\rangle=\langle\mathbf{t}, \mathbf{b}\rangle=0
$$

If $\alpha$ is pseudo null curve, the Frenet formulas have the form

$$
\left[\begin{array}{c}
\mathbf{t}^{\prime} \\
\mathbf{n}^{\prime} \\
\mathbf{b}^{\prime}
\end{array}\right]=\left[\begin{array}{ccc}
0 & \kappa & 0 \\
0 & \tau & 0 \\
-\kappa & 0 & -\tau
\end{array}\right]\left[\begin{array}{l}
\mathbf{t} \\
\mathbf{n} \\
\mathbf{b}
\end{array}\right],
$$

where the first curvature $\kappa=0$ if $\alpha$ is straight line, or $\kappa=1$ in all other cases. In this case, the following conditions are satisfied

$$
\langle\mathbf{t}, \mathbf{t}\rangle=\langle\mathbf{n}, \mathbf{b}\rangle=1,\langle\mathbf{n}, \mathbf{n}\rangle=\langle\mathbf{b}, \mathbf{b}\rangle=\langle\mathbf{t}, \mathbf{n}\rangle=\langle\mathbf{t}, \mathbf{b}\rangle=0 .
$$

If $\alpha$ is a null curve, the Frenet equations are given by

$$
\left[\begin{array}{c}
\mathbf{t}^{\prime} \\
\mathbf{n}^{\prime} \\
\mathbf{b}^{\prime}
\end{array}\right]=\left[\begin{array}{ccc}
0 & \kappa & 0 \\
\tau & 0 & -\kappa \\
0 & -\tau & 0
\end{array}\right]\left[\begin{array}{l}
\mathbf{t} \\
\mathbf{n} \\
\mathbf{b}
\end{array}\right],
$$

where the first curvature $\kappa=0$ if $\alpha$ is straight line, or $\kappa=1$ in all other cases. In particular, the following conditions hold:

$$
\langle\mathbf{t}, \mathbf{t}\rangle=\langle\mathbf{b}, \mathbf{b}\rangle=\langle\mathbf{t}, \mathbf{n}\rangle=\langle\mathbf{n}, \mathbf{b}\rangle=0,\langle\mathbf{n}, \mathbf{n}\rangle=\langle\mathbf{t}, \mathbf{b}\rangle=1 \text {. }
$$

\section{The Modified Orthogonal Frame with Curvature in Minkowski 3-Space}

Theorem 3.1. Let $\alpha$ and $\{\mathbf{t}, \mathbf{n}, \mathbf{b}\}$ be a unit speed curve in Minkowski 3-space and the Frenet frame along the curve, respectively. The relations between orthogonal frame $\{T, N, B\}$ and the classical Frenet frame $\{\mathbf{t}, \mathbf{n}, \mathbf{b}\}$ at non-zero points of $\kappa$ are

$$
T=\mathbf{t}, N=\kappa \mathbf{n}, B=\kappa \mathbf{b} .
$$

In this case, the following modified orthogonal frames hold: If $\alpha$ is timelike curve, then the orthogonal frame is

$$
\left[\begin{array}{c}
T^{\prime}(s) \\
N^{\prime}(s) \\
B^{\prime}(s)
\end{array}\right]=\left[\begin{array}{ccc}
0 & 1 & 0 \\
\kappa^{2} & \frac{\kappa^{\prime}}{\kappa} & \tau \\
0 & -\tau & \frac{\kappa^{\prime}}{\kappa}
\end{array}\right]\left[\begin{array}{c}
T(s) \\
N(s) \\
B(s)
\end{array}\right] .
$$

If $\alpha$ is a spacelike curve with a spacelike principal normal $\mathbf{n}$, then the the orthogonal frame is

$$
\left[\begin{array}{c}
T^{\prime}(s) \\
N^{\prime}(s) \\
B^{\prime}(s)
\end{array}\right]=\left[\begin{array}{ccc}
0 & 1 & 0 \\
-\kappa^{2} & \frac{\kappa^{\prime}}{\kappa} & \tau \\
0 & \tau & \frac{\kappa^{\prime}}{\kappa}
\end{array}\right]\left[\begin{array}{c}
T(s) \\
N(s) \\
B(s)
\end{array}\right] .
$$

If $\alpha$ is a spacelike curve with a spacelike binormal $\mathbf{b}$, then the orthogonal frame is

$$
\left[\begin{array}{c}
T^{\prime}(s) \\
N^{\prime}(s) \\
B^{\prime}(s)
\end{array}\right]=\left[\begin{array}{ccc}
0 & 1 & 0 \\
\kappa^{2} & \frac{\kappa^{\prime}}{\kappa} & \tau \\
0 & \tau & \frac{\kappa^{\prime}}{\kappa}
\end{array}\right]\left[\begin{array}{c}
T(s) \\
N(s) \\
B(s)
\end{array}\right] .
$$


If $\alpha$ is pseudo null curve, the modified frame is given by

$$
\left[\begin{array}{c}
T^{\prime}(s) \\
N^{\prime}(s) \\
B^{\prime}(s)
\end{array}\right]=\left[\begin{array}{ccc}
0 & 1 & 0 \\
0 & \frac{\kappa^{\prime}}{\kappa}+\tau & 0 \\
-\kappa^{2} & 0 & \frac{\kappa^{\prime}}{\kappa}-\tau
\end{array}\right]\left[\begin{array}{c}
T(s) \\
N(s) \\
B(s)
\end{array}\right] .
$$

If $\alpha$ is a null curve, the modified frame is given by

$$
\left[\begin{array}{c}
T^{\prime}(s) \\
N^{\prime}(s) \\
B^{\prime}(s)
\end{array}\right]=\left[\begin{array}{ccc}
0 & 1 & 0 \\
\kappa \tau & \frac{\kappa^{\prime}}{\kappa} & -\kappa \\
0 & -\tau & \frac{\kappa^{\prime}}{\kappa}
\end{array}\right]\left[\begin{array}{c}
T(s) \\
N(s) \\
B(s)
\end{array}\right]
$$

\section{The Modified Orthogonal Frame with Torsion in 3-Space}

Theorem 4.1. Let $\alpha$ and $\{\mathbf{t}, \mathbf{n}, \mathbf{b}\}$ be a unit speed curve in Minkowski 3-space and the Frenet frame along the curve, respectively. The relations between orthogonal frame $\{T, N, B\}$ and the classical Frenet frame $\{\mathbf{t}, \mathbf{n}, \mathbf{b}\}$ at non-zero points of $\tau$ are

$$
T=\mathbf{t}, N=\tau \mathbf{n}, B=\tau \mathbf{b},
$$

where

$$
\begin{gathered}
\langle T, T\rangle=1,\langle N, N\rangle=\langle B, B\rangle=\tau^{2}, \\
\langle T, N\rangle=\langle T, B\rangle=\langle N, B\rangle=0 .
\end{gathered}
$$

In this case, the following modified orthogonal frame hold:

$$
\left[\begin{array}{l}
T^{\prime}(s) \\
N^{\prime}(s) \\
B^{\prime}(s)
\end{array}\right]=\left[\begin{array}{ccc}
0 & \frac{\kappa}{\tau} & 0 \\
-\kappa \tau & \frac{\tau^{\prime}}{\tau} & \tau \\
0 & -\tau & \frac{\tau^{\prime}}{\tau}
\end{array}\right]\left[\begin{array}{c}
T(s) \\
N(s) \\
B(s)
\end{array}\right] .
$$

Theorem 4.2. Let $\alpha$ and $\{\mathbf{t}, \mathbf{n}, \mathbf{b}\}$ be a unit speed curve in Minkowski 3-space and the Frenet frame along the curve, respectively. The relations between orthogonal frame $\{T, N, B\}$ and the classical Frenet frame $\{\mathbf{t}, \mathbf{n}, \mathbf{b}\}$ at non-zero points of $\tau$ are

$$
T=\mathbf{t}, N=\tau \mathbf{n}, B=\tau \mathbf{b} .
$$

In this case, the following modified orthogonal frames hold: If $\alpha$ is timelike curve, then the orthogonal frame is

$$
\left[\begin{array}{c}
T^{\prime}(s) \\
N^{\prime}(s) \\
B^{\prime}(s)
\end{array}\right]=\left[\begin{array}{ccc}
0 & \frac{\kappa}{\tau} & 0 \\
\kappa \tau & \frac{\tau^{\prime}}{\tau} & \tau \\
0 & -\tau & \frac{\tau^{\prime}}{\tau}
\end{array}\right]\left[\begin{array}{c}
T(s) \\
N(s) \\
B(s)
\end{array}\right] .
$$

If $\alpha$ is a spacelike curve with a spacelike principal normal $\mathbf{n}$, then the the orthogonal frame is

$$
\left[\begin{array}{c}
T^{\prime}(s) \\
N^{\prime}(s) \\
B^{\prime}(s)
\end{array}\right]=\left[\begin{array}{ccc}
0 & \frac{\kappa}{\tau} & 0 \\
-\kappa \tau & \frac{\tau^{\prime}}{\tau} & \tau \\
0 & \tau & \frac{\tau^{\prime}}{\tau}
\end{array}\right]\left[\begin{array}{c}
T(s) \\
N(s) \\
B(s)
\end{array}\right] .
$$

If $\alpha$ is a spacelike curve with a spacelike binormal $\mathbf{b}$, then the orthogonal frame is

$$
\left[\begin{array}{c}
T^{\prime}(s) \\
N^{\prime}(s) \\
B^{\prime}(s)
\end{array}\right]=\left[\begin{array}{ccc}
0 & \frac{\kappa}{\tau} & 0 \\
\kappa \tau & \frac{\tau^{\prime}}{\tau} & \tau \\
0 & \tau & \frac{\tau^{\prime}}{\tau}
\end{array}\right]\left[\begin{array}{c}
T(s) \\
N(s) \\
B(s)
\end{array}\right]
$$

If $\alpha$ is pseudo null curve, the modified frame is given by

$$
\left[\begin{array}{c}
T^{\prime}(s) \\
N^{\prime}(s) \\
B^{\prime}(s)
\end{array}\right]=\left[\begin{array}{ccc}
0 & \frac{\kappa}{\tau} & 0 \\
0 & \frac{\tau^{\prime}}{\tau}+\tau & 0 \\
-\kappa \tau & 0 & \frac{\tau^{\prime}}{\tau}-\tau
\end{array}\right]\left[\begin{array}{c}
T(s) \\
N(s) \\
B(s)
\end{array}\right]
$$


If $\alpha$ is a null curve, the modified frame is given by

$$
\left[\begin{array}{c}
T^{\prime}(s) \\
N^{\prime}(s) \\
B^{\prime}(s)
\end{array}\right]=\left[\begin{array}{ccc}
0 & \frac{\kappa}{\tau} & 0 \\
\tau^{2} & \frac{\tau^{\prime}}{\tau} & -\kappa \\
0 & -\tau & \frac{\tau^{\prime}}{\tau}
\end{array}\right]\left[\begin{array}{c}
T(s) \\
N(s) \\
B(s)
\end{array}\right]
$$

\section{References}

[1] https://en.wikipedia.org/wiki/Frenet\%E2\%80\%93Serret_formulas

[2] J. Walrave, Curves and surfaces in Minkowski space, Doctoral thesis, K. U.Leuven, Faculty of Science, Leuven (1995)

[3] K. Ilarslan, Spacelike Normal Curves in Minkowski Space $E_{1}^{3}$,Turk J Math 29 (2005) , 53-63.

[4] M. Grbovic, K.Ilarslan,E.Nesovic,On null and pseudo null Mannheim curves in Minkowski 3-space, J. Geom. 105 (2014), 177-183.

[5] M. K. Karacan,Y.Yayli,On the Geodesics of Tubular Surfaces in Minkowski 3-Space,Bull. Malays. Math. Sci. Soc. (2) 31 (1) (2008), 1-10.

[6] M. Petrovic-Torgasev,E. Sucurovic, Some characterizations of Lorentzian spherical pacelike curves with the timelike and null principal normal, Mathematica Moravica 4 (2000), 83-92.

[7] M. Petrovic-Torgasev, E. Sucurovic, Some characterizations of curves lying on the pseudohyperbolic space $H_{0}^{2}$ in the Minkowski space $E_{1}^{3}$, Kragujevac J. Math., 22 (2000),71-82.

[8] T. Sasai, The Fundamental Theorem of Analytic Space Curves And Apparent Singularities of Fuchsian Differential Equations,Tohoku Math. Journ., 36 (1984),17-24.

\section{Affiliations}

\section{BAHADDIN BUKCU}

AdDress: Gazi Osman Pasa University, Faculty of Sciences and Arts, Department of Mathematics, 60250, TokatTURKEY

E-MAIL: bbukcu@yahoo.com

MURAT KeMAL KARACAN

AdDRESS: Usak University, Faculty of Sciences and Arts, Department of Mathematics,1 Eylul Campus, 64200,UsakTURKEY

E-MAIL: murat.karacan@usak.edu.tr 